\title{
Autotrascendencia y espiritualidad en personas adultas mayores, reflexión para el cuidado ${ }^{1}$ Self-transcendence and spirituality in old age, reflection for care Auto-transcendência e espiritualidade na velhice, reflexão para o cuidado
}

Alma Cristina Alcocer Sosa ${ }^{2}$, Patricia Cid Henriquez ${ }^{3}$, Raúl Fernando Guerrero-Castañeda ${ }^{4}$

\section{RESUMEN}

Objetivo: Reflexionar sobre la autotrascendencia y la espiritualidad en personas adultas mayores. Método: Se trata de un ensayo reflexivo sobre la autotrascendencia y la espiritualidad en personas adultas mayores para su aplicación en el cuidado de enfermería. Se revisó la teoría de autotrascendencia y se relacionó con la espiritualidad y el cuidado a la persona adulta mayor. Resultado: Se obtuvieron dos temas principales. El fenómeno de autotrascendencia y la espiritualidad. Asimismo, la espiritualidad en el cuidado de enfermería a la persona adulta mayor para la autotrascendencia. Esta última expone una cualidad para integrar la vida. Además, al ser la espiritualidad un medio para lograr este proceso y ser promotor del bienestar y la salud en esta población, las enfermeras en el cuidado pueden promoverla. Conclusión: El camino espiritual ayuda a comprender la vida. Es posible establecer para la persona adulta mayor una relación entre la autotrascendencia, el cuidado y el bienestar en la vejez.

Descriptores: Atención de enfermería; Enfermería; Espiritualidad; Anciano (DeCS)

\footnotetext{
${ }^{1}$ Fecha de recepción: 21 de febrero del 2020

Fecha de aceptación: 25 de octubre del 2020

${ }^{2}$ Enfermera. Estudiante de la Maestría en Ciencias de Enfermería. Universidad de Guanajuato. México. E-mail: almaalcocer842@gmail.com ORCID: https://orcid.org/0000-0003-1641-6572

${ }^{3}$ Enfermera- Profesor Titular, Directora Programa Magister en Enfermería, Facultad de Enfermería, Universidad de Concepción. Email: patcidh@gmail.com ORCID: https://orcid.org/0000-0002-2821-0558

${ }^{4}$ Enfermero. Doctor en Ciencias de Enfermería. Profesor de la Universidad de Guanajuato. México. Email: ferxtom@hotmail.com ORCID: https://orcid.org/0000-0003-3996-5208
} 
ABSTRACT

\begin{abstract}
Objective: To reflect on self-transcendence and spirituality in the elderly. Method: This is a reflective essay on self-transcendence and spirituality in the elderly for application in nursing care. The theory of self-transcendence was reviewed in relation to spirituality and elder care. Result: Two main themes were obtained: The phenomenon of selftranscendence and spirituality, and Spirituality in nursing care of the elderly for self-transcendence.
\end{abstract}

Self-transcendence exhibits a quality for integrating life, with spirituality being one means of achieving this process and being a promoter of well-being and health in the elderly, nurses in care can promote spirituality. Conclusion: The spiritual path helps to understand life, it is possible to establish its relationship with self-transcendence in the elderly, care and well-being in old age.

Keyword: Nursing care; Nursing; Spirituality; Aged (DeCS)

\section{RESUMO}

Objetivo: Refletir sobre a autotranscendência e a espiritualidade nos idosos. Método: Este é um ensaio reflexivo sobre autotranscendência e espiritualidade nos idosos para aplicação em cuidados de enfermagem. A teoria da autotranscendência foi revista em relação à espiritualidade e ao cuidado do idoso. Resultado: Dois temas principais foram obtidos: o fenômeno da autotranscendência e da espiritualidade, e a Espiritualidade no cuidado dos idosos para a autotranscendência. A autotranscendência exibe uma qualidade para integrar a vida, sendo a espiritualidade um meio para alcançar este processo e para ser um promotor de bem-estar e saúde nos idosos, os enfermeiros no atendimento podem promover a espiritualidade. Conclusão: $O$ caminho espiritual ajuda a compreender a vida, é possível estabelecer sua relação com a autotranscendência nos idosos, o cuidado e o bemestar na velhice.

Palabras Chave : Cuidados de enfermagem; Enfermagem; Espiritualidade; Idoso (DeCS) 


\section{INTRODUCCIÓN}

Las teorías de enfermería bajo el enfoque humanista han destacado la importancia de cuidar a la persona en todas sus dimensiones. En general, algunos investigadores han observado que en la mayoría de los casos el cuidado se centra en el cuerpo, luego se abordan los aspectos sociales y psicológicos y, en menor medida, la espiritualidad.

Quizá esta poca valorización se deba a elementos contextuales y culturales disciplinares, por ejemplo, la falta de desarrollo de competencias para el cuidado espiritual tanto en la formación como en el actuar profesional. Sin embargo, se torna necesario abordar el cuidado de la espiritualidad por su significado para la persona adulta mayor.

El aumento de la población mundial de adultos mayores permite reconocer la importancia del cuidado en toda la población. De acuerdo con las características de la vejez, se muestra un aumento en la necesidad espiritual ${ }^{1}$, ya que las personas suelen acercarse más a esta esfera durante el proceso de envejecimiento ${ }^{2}$. Por ello, los cuidados deben enfocarse en todos los aspectos de la persona, tanto biológicos, psicológicos y sociales como espirituales.

La espiritualidad actúa como un pilar en la persona mayor para afrontar los cambios y pérdidas que se viven en esta etapa, además proporciona un consuelo y alivio ante situaciones que quizá no se pueden modificar ${ }^{3}$. Considerando al ser humano como un todo único y pensando en potenciar sus capacidades, las personas profesionales en enfermería han teorizado sobre el cuidado y han aportado elementos para su comprensión, incluida la espiritualidad.

La teoría de la autotrascendencia fue creada bajo la perspectiva de procesos de salud y desarrollo. En esta, la persona adulta mayor valora su vida a pesar de las dificultades en las que se encuentre. Asimismo, los cambios atribuidos al proceso de envejecimiento en todas las áreas contribuyen a un cambio de pensamiento en donde las personas mayores experimentarían un estado de bienestar, enfatizando la vejez como el final de la vida y favoreciendo el sentido de conexión intra, inter y transpersonal ${ }^{2}$. A diferencia de la autorrealización, donde esta última etapa es un fin, la autotrascendencia es un proceso de conciencia sobre el mundo y sobre el ser en el mundo, más allá del propio yo, lo que hace que la vida tenga un sentido y propósito $^{5-6}$. Por ello, la autotrascendencia en la vejez se relaciona con un sentido del todo unificado en el ser persona adulta mayor y sus más profundos valores humanos.

La vejez se caracteriza por una serie de situaciones que desafían el sentido de vida y la espiritualidad viene a dar una visión integradora de la vida y la muerte como un fenómeno de sabiduría que enaltece al ser humano ${ }^{7}$, que le ayuda a afrontar las situaciones cotidianas y puede favorecer el sentido de autotrascendencia.

Considerando que la población adulta mayor aumenta y hay un aumento considerable de la espiritualidad en esta etapa $^{8}$, esta puede favorecer una vejez satisfactoria aun en condiciones de institucionalización ${ }^{9}$, pues aporta elementos que permiten comprender el fenómeno de autotrascendencia en la vejez como una forma de comprensión del mundo desde un sentido de plenitud en esta etapa de desarrollo ${ }^{10}$. Por lo anterior, el objetivo de este documento es elaborar una reflexión sobre la autotrascendencia y la espiritualidad en personas adultas mayores. 


\section{DESARROLLO}

\section{El fenómeno de autotrascendencia y la espiritualidad}

Se entiende la autotrascendencia como constructo que supone una forma madurativa, es decir, las personas tienen un potencial de cambio, un proceso de maduración cognitiva que en etapas posteriores de desarrollo permeará la resolución de conflictos ${ }^{11}$.

Por lo tanto, la vejez es vista como una etapa de sabiduría donde los significados de la vida adquieren una forma visionaria del mundo y del universo como un todo, integrando las experiencias positivas y negativas para conformar el yo presente. Este se configura como una triada entre la comprensión del pasado, el afrontamiento del presente y las esperanzas del futuro.

La autotrascendencia favorece el bienestar ${ }^{12}$. Una persona adulta mayor autotrascendente expresará una sensación de bienestar consigo misma y con el mundo que le rodea, como una conciencia que se vive de manera cotidiana en la búsqueda de sentido. El ser humano no es un ser limitado a necesidades, sino un ser pandimensional, sin límites e integrado como un todo ${ }^{1}$.

La autotrascendencia evoca una forma de pensamiento de plenitud, integridad y coherencia alcanzado en la vejez. Se destaca, precisamente, la sabiduría, el acercamiento con la muerte que dota al ser humano de una conciencia de su unión con el cosmos y su sentido de ser humano, una noción de cooperación y solidaridad con los demás, una apertura al mundo y a sus formas de aprendizaje, además de una conexión espiritual, un conjunto de formas subjetivas de percibir el mundo ${ }^{1-2}$.

La persona adulta mayor al enfrentarse a los cambios en su ciclo vital mueve sus formas cotidianas, reflexiona sobre su posición en el momento, hace uso de los medios que dispone para afrontarlas y es capaz de encontrar el sentido de lo vivido; la espiritualidad es uno de esos recursos ${ }^{11}$.

La autotrascendencia es una búsqueda de sentido mediante un camino espiritual, un encuentro consigo mismo, con los demás, con el entorno o bien con una divinidad ${ }^{12}$. Sobrepasa el romanticismo, pues la persona intenta por estos medios encontrar un equilibrio que le permita estar orientada en un propósito de vida aun y cuando ese propósito sea la muerte inminente. Es la búsqueda de estar en paz y de aceptar esa muerte para irse en paz y tranquilidad. Lo importante es la propia persona adulta mayor en contacto consigo mismo, con lo que la rodea y dando significado a su vida.

El objetivo de una vejez satisfactoria es una sensación de bienestar integrada del yo como parte del todo, un sentimiento de salud integral donde el autoconcepto positivo, felicidad, moralidad, forman parte del crecimiento espiritual y, por lo tanto, se promueve la autotrascendencia, favoreciendo ese sentido de vida ${ }^{13}$.

La persona adulta mayor, en esta mirada, ha atravesado por una serie de dificultades que desafían su autoconcepto. Sumado a ello, la salida de los hijos o las hijas de casa, la jubilación, la viudez, las pérdidas de personas cercanas y familiares, las pérdidas cognitivas y emocionales, así como el propio rechazo de la sociedad ${ }^{14}$ son solo algunas de las situaciones que se han afrontado y de las cuales se espera haya una integración de aprendizajes que conduzcan a una sabia forma de envejecer. 
Todas estas situaciones podrían situarse como formas de vulnerabilidad. La persona adulta mayor en cada uno de esos eventos puede verse desafiado en su propio concepto. Se da una conciencia de la mortalidad personal en el sentido que da cuenta de su capacidad de afrontarlas. Por lo tanto, la vulnerabilidad se relaciona con sucesos que hacen consciente a la persona de la fragilidad propia y ajena, que si no hunden al individuo, lo motivan para que salga hacia delante y sobrevenga de esa dificultad con un nuevo pensamiento de crecimiento personal, propiciado por ese momento de conciencia sobre la muerte ${ }^{1}$.

Estos cambios en la persona adulta mayor pueden no ser visibles a quienes les rodean, sino que se relacionan más bien con el sentimiento interno y suelen ser expresados por la persona mayor. Cada uno no debe ser en sí un evento negativo, precisamente la subjetividad de las situaciones y experiencias vividas por cada persona a lo largo de su ciclo vital está íntimamente relacionada con sus significados y los aprendizajes.

Si hasta este punto se consideran los eventos de la vida como potenciales situaciones de vulnerabilidad para la persona mayor, la autotrascendencia a su vez se relacionaría directa y positivamente con el bienestar en la vejez ${ }^{15-}$ ${ }^{16}$. Reed menciona la influencia de factores contextuales y personales que interfieren de manera positiva o negativa en la autotrascendencia de las personas ${ }^{2}$, uno de estos factores positivos es la espiritualidad.

La espiritualidad es esencial en las personas para lograr la salud y bienestar ${ }^{17}$. Se le considera una cualidad de nuestra especie que es desarrollada individualmente. Su desarrollo permite que la persona sea capaz de lograr sus propósitos de vida con pleno sentido de su existencia. Sin embargo, las personas que no la desarrollan o lo hacen de forma incompleta podrían tener mayores problemas para lograr satisfacción en su vida ${ }^{18}$.

El concepto de espiritualidad ha sido de interés para varias disciplinas debido a la repercusión que tiene sobre la salud de las personas. Desde el punto de vista psicológico, la espiritualidad puede verse desde una perspectiva religiosa o humanista, pues está conectado con lo sobrenatural místico y se extiende más allá de la religión organizada ${ }^{19}$.

La espiritualidad puede estar relacionada con la religión. Es en esta donde regularmente las personas tienen su primer encuentro con lo divino, por lo que los conceptos han sido utilizados como iguales. La religión está basada en construcciones sociales que generalmente obedecen a alguna congregación o culto, mientras que la espiritualidad se relaciona con el recurso interno de la persona, que incluyen sensaciones y sentimientos de un poder más allá de la comprensión humana ${ }^{19-20}$. La espiritualidad puede ser desarrollada incluso fuera de la religión, es de carácter dinámico.

La espiritualidad sigue siendo un concepto abstracto de índole subjetivo, sin embargo, existe una variedad de escalas que pretenden, bajo una mirada teórica particular, acercarse a su medición de forma objetiva. Una revisión mostró un total de 23 escalas para medir la espiritualidad ${ }^{21}$, las cuales, pese a los niveles altos de confiabilidad demostrados, no dejan de presentar un sesgo importante: cada persona tiene percepciones distintas de su espiritualidad y la forma en que alguien la perciba o la manifieste puede ser muy variable.

En algunos estudios se ha demostrado la espiritualidad como soporte, que alivia la ansiedad y estrés ${ }^{19}$. Esto parece ser mayor en las personas de edad avanzada ${ }^{22}$. La persona adulta mayor se encuentra en la última etapa de su vida 
y tiene un encuentro más próximo con la muerte, por lo que la espiritualidad refuerza su sentido de conexión con aquello que le deviene; es una forma de autotrascendencia integrada.

\section{Espiritualidad en el cuidado de enfermería a la persona adulta mayor para la autotrascendencia}

La espiritualidad como un factor personal para la autotrascendencia aparece con mayor frecuencia en literatura con personas adultas mayores. Esto podría explicarse porque las personas mayores presentan mayores niveles de espiritualidad y, por lo tanto, de autotrascendencia.

La espiritualidad tiene un peso importante en la atención y sentimiento de satisfacción en las personas. Integra la contemplación y la reflexión sobre la existencia, asimismo, es algo que se pone a prueba en algunos eventos de la vida. Se comporta como una herramienta de ayuda y consuelo, sin embargo, a pesar de ser un aspecto importante dentro de la salud de la persona, suele ser poco abordada en el cuidado de enfermería ${ }^{23}$.

Partiendo de la reflexión del ciclo vital, desde la niñez se suelen hacer proyecciones hacia lo que se desea en la vida adulta, se considera como una meta personal tener una carrera o familia, un patrimonio o un trabajo bien establecido; sin embargo, no suele visualizarse la vejez como una meta. Si durante la juventud y la adultez se lograron los proyectos de vida es probable que durante la etapa de vejez se busque más estar en armonía y tranquilidad consigo mismo, su familia y la sociedad. En cambio, si durante alguna de las etapas de vida previas no se logró satisfacer alguna de las metas básicas, se puede generar insatisfacción o mala adaptación a la vejez.

En la vejez se logra alcanzar una madurez íntegra, abierta a nuevos horizontes espirituales, con una mirada a autotrascender. Se suele tener más tiempo para reflexionar sobre el sentido de la vida y así, la espiritualidad se vuelve un aspecto importante en la vejez.

La espiritualidad en las personas mayores suele ser alta, se asocia a resiliencia, bienestar y satisfacción de la vida, es un pilar para afrontar experiencias negativas ${ }^{24-26}$, además de asociarse a una mejor adaptación en el proceso de envejecimiento. Algunas personas mayores ven la espiritualidad como una forma de conectarse con un ser superior para pedir las cosas que no pueden realizar o no pueden aceptar en el proceso de envejecimiento. Los temas más relevantes en este sentido son las pérdidas a las que se enfrentan, pues la espiritualidad es una forma de encontrar consuelo ${ }^{24}$.

La espiritualidad también promueve la motivación para autocuidarse, establecer y cumplir metas, tener expectativas y disfrutar del entorno familiar y social, lo que permite afrontar los desafíos que se le presenten hasta que llegue la hora de la muerte.

La espiritualidad se manifiesta de distintas maneras entre las personas adultas mayores, pues la situación de vida, sus creencias, costumbres y tradiciones influye en la forma en que se vive la espiritualidad. Algunas manifestaciones están relacionadas a la religiosidad como asistir a una iglesia o pertenecer a un grupo religioso y otras más relacionados a la sensación de confianza y vehemencia en un ser superior ${ }^{11}$. 
En este punto, la espiritualidad en sus manifestaciones significativas y personales viene a conectar a la persona adulta mayor con su vida, integrando el sentido de ser en el universo y su misión en el mundo. Uno de los factores personales que favorecen la autotrascendencia ${ }^{14}$, además de ser contextual, en la persona adulta mayor, se relacionan con las creencias y prácticas religiosas de forma más común.

El ser conscientes de la importancia que tiene la espiritualidad en la última etapa de la vida permite crear nuevas formas de fomentar el cuidado en la persona mayor, al respetar las creencias espirituales y permitir su manifestación.

A través de la espiritualidad, se puede ver de manera objetiva las situaciones del pasado que han llevado a la persona a estar donde está ahora y que, de alguna u otra forma, influye en donde estará después. Lo anterior, porque la persona cree que todas las situaciones que ha pasado tienen un por qué y un para qué, es decir, no son aisladas, sino que cada una tiene un espacio particular en la vida. La autotrascendencia se considera la cúspide de lo espiritual, expresa la conexión con todo, por lo que, como se ha mencionado, dota a las personas de sentido no solo en situaciones específicas, sino de toda su existencia.

Dentro de la promoción de la salud, las enfermeras podrían buscar facilitar los medios para que la persona haga uso de diversas estrategias para encontrar ese equilibrio vital. Podría parecer que no es parte del cuidado, pero la esencia del cuidado de enfermería debe contemplar también el factor espiritual como parte del cuidado holístico de la enfermera ${ }^{29}$, pues promueve la salud en consonancia con el bienestar.

La espiritualidad en el cuidado de enfermería parece ser invisible aun y cuando se ha contemplado en el fundamento disciplinar, de acuerdo con una investigación, se aprecia que ni el personal de salud está capacitado para abordar temas espirituales ni los espacios de las instituciones de salud son los adecuados para crear ese vínculo y que el paciente esté en contacto con su espiritualidad. En los cuidados pocas veces se contemplan los aspectos espirituales, la atención se centra en alteraciones físicas ${ }^{27}$.

Las enfermeras requieren buscar y vivir su propia espiritualidad, así como la capacitación en el tema que permita tener una apertura más allá de la religiosidad propia, además, un sentido de comprensión de las creencias y prácticas de fe y esperanza de las personas cuidadas.

Una investigación señala que pacientes refieren como necesidad espiritual el que algún sacerdote los visite cuando se encuentran enfermos de muerte. Asimismo, que la enfermera dedique un poco de su tiempo para meditar o hablar sobre temas o aspectos espirituale ${ }^{20}$. Estudios como este podrían ser la clave para abordar el tema de la espiritualidad e involucrar a las personas en el cuidado de su propia salud. La persona profesional en enfermería puede tomar el tiempo para escuchar a sus pacientes, acercárseles y estar presente no sólo física sino espiritualmente. Las personas admiten la presencia de la persona profesional en enfermería como la proveedora de cuidado y su interacción como seres humanos extiende la noción de la autotrascendencia, juntas aprenderían en el proceso de cuidar.

La espiritualidad es un indicador empírico de la capacidad de las personas para la autotrascendencia, pues es una forma de interacción con algo más elevado que el propio ser, un punto de divinidad y de comprensión de la vida ${ }^{23}$. De ahí la importancia de su comprensión como fenómeno relacionado con la salud, si la espiritualidad es un factor 
para la autotrascendencia de las personas mayores, el fomentar las prácticas y creencias espirituales podría ayudar como una manera de aproximación con la enfermería.

Desde esta dinámica la espiritualidad se convierte en un punto de intervención para las personas profesionales en esta área ${ }^{4}$, pues es un medio que la persona toma para afrontar situaciones difíciles o bien como un punto de equilibrio en la vida. Por tal motivo, esto remite al carácter subjetivo de su valoración en las personas mayores, contemplando sus significados, formas de manifestación y sentido para la vida misma.

Se sabe que las personas profesionales en enfermería se enfrentan en el cuidado a situaciones relacionadas con la espiritualidad y, en muchas ocasiones, no están del todo capacitadas para abordarlas ${ }^{27-29}$. La vivencia de la propia espiritualidad y el sentido de ser en su acción cotidiana del cuidado puede sentar las bases para la reflexión y abrir el camino para abordar estos aspectos subjetivos, ya que sí se tiene evidencia de sus beneficios en el estado de salud.

Desde el cuidado de enfermería a la persona adulta mayor, el objetivo es promover medios para favorecer la autotrascendencia por medio de la espiritualidad desde la vivencia propia, por un lado, de cada persona mayor en su integración vital y, por otro, en la situación que vive actualmente. Por ejemplo, comunitariamente, se puede hacer promoción de los beneficios que aporta la espiritualidad para el bienestar durante la hospitalización en la manifestación de las prácticas y creencias, según las necesidades de cada persona adulta mayor.

La autotrascendencia como un proceso de autoconocimiento y de renovación del espíritu y de la vida misma, a pesar de ser un fenómeno subjetivo, puede ser una fuente de reflexión para las personas profesionales en enfermería, pues en el cotidiano del cuidado se viven situaciones que requieren de esa reflexividad en el afrontamiento de situaciones de enfermedad, muerte, dolor y sufrimiento. Estos son momentos en los cuales la persona profesional en enfermería puede acompañar en la dificultad y ir más allá de la práctica de tratamientos y medicaciones para ayudar a encontrar el propósito de lo vivido. El encuentro con la persona adulta mayor, no solo en estos tratamientos, sino en el conocimiento pleno de su búsqueda de sentido vital, ayudaría a las enfermeras a integrar el cuidado espiritual para favorecer esta conexión.

Dentro de las limitaciones de la presente reflexión destaca que debido a su naturaleza no es posible ahondar en las temáticas abordadas, sin embargo, se presentan y se ponen sobre la discusión para su análisis y profundización en el cuidado cotidiano de enfermería.

El aporte de esta reflexión para la enfermería es sobre aspectos que, si bien resultan subjetivos para algunas posturas rígidas, también invitan a una postura pragmática de la incorporación de las teorías de enfermería para sustentar el cuidado.

\section{CONCLUSIONES}

La autotrascendencia es un movimiento personal, una visión más allá de las situaciones presentes que le permite a cada persona adulta mayor buscar estrategias para lograr un equilibrio consigo misma en el mundo y con el mundo. La espiritualidad es un factor que puede mediar esta búsqueda y que dota de un sentido de existencia, una razón del 
momento y de su afrontamiento. Este fenómeno se incrementa con la edad y pone de manifiesto la importancia de esta dimensión para las personas.

La teoría de autotrascendencia abre el camino a las personas profesionales en enfermería a explorar a las personas más allá de necesidades básicas. A través del cuidado y del cuidado espiritual, se contribuye al desarrollo de las personas adultas mayores y al afrontamiento de sus momentos difíciles, además de promover la salud y el bienestar.

\section{REFERENCIAS BIBLIOGRÁFICAS}

1. Zanardini FEH. A espiritualidade em uma sociedade que envelhece. Caminhos. 2020;18(1): 94-105. DOI: https://doi.org/10.18224/cam.v18.n1.7731

2. Whitney G. Understanding the Presence of Gerotranscendence Among Older Adults. Adulspan Journal. 2018;17(1):27-40. DOI: https://doi.org/10.1002/adsp.12051

3. Reis LA, Menezes TMO. Religiosidade e espiritualidade nas estratégias de resiliência do idoso longevo no cotidiano. Rev Bras Enferm.2017;70(4):761-766. DOI: http://dx.doi.org/10.1590/0034-7167-2016-0630.

4. Reed PG. Pamela G. Reed: Self-Transcendence Theory. En: Raile-Alligood M. Nursing Theorist and their work. Missouri: Elsevier; 2018. p. 463-476.

5. Rusu M. The Process of Self-Realization-From the Humanist Psychology Perspective. Psychology. 2019; 10: 1095-1115. DOI: https://doi.org/10.4236/psych.2019.108071.

6. Santos MA dos. Corpo envelhece, espírito transcende: qual a contribuição da espiritualidade para a subjetividade do idoso? Pretextos. 2020; 5(9): 260-74. Disponible http://periodicos.pucminas.br/index.php/pretextos/article/view/22275

7. Haynes C. Identity, transcendence and the true self: Insights from psychology and contemplative spirituality. Herv. teol. stud. 2016; 72(4): 1-9. DOI: http://dx.doi.org/10.4102/hts.v72i4.3455.

8. Grangeiro AFB, Gomes LO, Alves VP, Faleiros VP. Impacto da religiosidade e espiritualidade em pessoas idosas centenárias: revisão sistemática. Ciências Sociais e Religião. 2017; 19(27): 173-182. Disponible en: https://econtents.bc.unicamp.br/inpec/index.php/csr/article/view/12506/7868

9. Miranda RFNA, Pereira ER, Silva RMCRA, Días FA. Sentido da vida e espiritualidade em idosos institucionalizados. Res., Soc. Dev. 2020; 9(6): e72962799. DOI: http://dx.doi.org/10.33448/rsd-v9i6.2799

10. Bittencourt MN, Marques MID, Barroso TMMDA. Contributos das teorias de enfermagem na prática da promoção de saúde mental. Rev. Enf. Ref. 2018; 4(18): 125-132. DOI: http://dx.doi.org/10.12707/RIV18015.

11. Thiengo PCS, Gomes AMT, Mercês MC, Couto PLS, França LCM, Silva AB. Espiritualidade e religiosidade no cuidado em saúde: revisão integrativa. Cogitare Enferm. 2019; $24 . \quad$ DOI: http://dx.doi.org/10.5380/ce.v24i0.58692. 
12. Guerrero-Castañeda RF, Ojeda-Vargas MG. Análisis del concepto de Autotrascendencia. Importancia en el cuidado de enfermería al adulto mayor. Cult. Cuid. 2015; 19(42): 26-37. DOI: https://doi.org/10.14198/cuid.2015.42.05

13. Fiske EA. Self-Transcendence, Well-Being, and Vulnerability in Healthcare Mission Participants. Nurs Sci Q. 2019; 32 (4):306-13. DOI: https://doi.org/10.1177\%2F0894318419864326

14. Robledo CA, Orejuela JJ. Teorías de la sociología del envejecimiento y la vejez. Guillermo de Ockham.2020; 18(1):95-102. DOI: https://doi.org/10.21500/22563202.4660

15. Ardelt M, Gerlach KR, Vaillant GE, Early and Midlife Predictors of Wisdom and Subjective Well-Being in Old Age. J Gerontol. 2018; 73(8): 1514-1525, https://doi.org/10.1093/geronb/gby017

16. McCarthy VL, Hall LA, Crawford TN. Facilitating Self-Transcendence: An Intervention to Enhance WellBeing in Late Life. West. J. Nurs. Res. 2018; 40(6): 854-873. DOI: https://doi.org/10.1177\%2F019394591769073111

17. Reed PG. An emerging paradigm for the investigation of spirituality in nursing. RINAH. 1992;15(5):349-57 DOI: https://doi.org/10.1002/nur.4770150505

18. Bugajska BE. The ninth stage in the cycle of life - reflections on E. H. Erikson's theory. Ageing Soc. 2017; 37(6): 1095-1110. DOI: https://doi.org/10.1017/S0144686X16000301

19. Kumari C, Pandey R, Srivastava AK. Role of Religion and Spirituality in Strees Management Among Nurses. Psychol. Stud. 2018; 63:187-199. DOI: https://doi.org/10.1007/s12646-018-0454-x

20. Withers A, Zuniga K, Van Sell SL. Spirituality: Concept Analysis. Int J Nurs Clin Pract. 2017; 4: 234. DOI: https://doi.org/10.15344/2394-4978/2017/234

21. Sieverson, C., Evans, V., Florenzano, R., \& Fernández, L ¿Qué elementos se incorporan en las evaluaciones de la espiritualidad y religiosidad? Una revisión de instrumentos validados. Rev GPU. 2016; 12; 2: $216-223$. Disponible en: http://revistagpu.cl/2016/GPU_junio_2016_PDF/INV_Que_elementos.pdf

22. Haugan G, Kuven BM, Eide WM, Taasen SE, Rinnan E, Wu VX, Drageset J, André B. Nurse-patient interaction and self-transcendence: assets for a meaningful life in nursing home residents?. BMC Geriatr. 2020; 20:168. DOI: https://doi.org/10.1186/s12877-020-01555-2

23. Musa AS, Al Qadire MI, Aljezawi M, Tawalbeh L, Aloush S, Albanian FZ. Barriers to the Provision of Spiritual Care by Nurses for Hospitalized Patients in Jordan. Res Theory Nurs Pract. 2020; 33(4). DOI: https://doi.org/10.1891/1541-6577.33.4.392

24. Guerrero-Castañeda RF, Menezes TMO, Prado ML, Galindo-Soto JA. Espiritualidad y religiosidad para la trascendencia del ser anciano. Rev. Bras. Enferm. 2019; 72(Suppl 2): 259-265. DOI: https://doi.org/10.1590/0034-7167-2018-0840. 
25. Scortegagna H, Pichler N, Fáccio L. The experience of spirituality among institutionalized elderly people. Rev. Bras. Geriatr. Gerontol. 2018; 21(3):304-311. DOI: http://dx.doi.org/10.1590/1981-22562018021.180011

26. Santos EL, Navarine TCRR, Costa MML. Espiritualidade, enfermagem e saúde do idoso: cuidando da integralidade do ser. Nursing. 2018; 21(245):2401-2403. Disponible en: http://www.revistanursing.com.br/revistas/245/pg38.pdf

27. Kroning M. Student Perceptions of Spirituality and Spiritual Care. J Christ Nurs. 2018; 35(2): E17-E20. DOI: https://doi.org/10.1097/CNJ.0000000000000490

28. Timmins F, Caldeira S. Understanding spirituality and spiritual care in nursing. Nurs Stand. 2017; 31(22):5057. DOI: 18. Reed PG. Spirituality and well-being in terminally ill hospitalized adults. RINAH. 1987 Oct;10(5):335-44. DOI: https://doi.org/10.7748/ns.2017.e10311

29. Atashzadeh-Shoorideh F, Zakaryaee NS, Fani M. The barriers and facilitators in providing spiritual care for parents who have children suffering from cancer. J Family Med Prim Care. 2018;7(6):1319-1326. DOI: https://doi.org/10.4103/jfmpc.jfmpc 76 _ 18 